\title{
La emersión como transcodificación del espacio digital global al espacio local físico.
}

\section{Emersion as a transcodification from the digital global to the local physical space.}

\author{
TIPO DE TRABAJO:
}

Comunicación.

PALABRAS CLAVE:

Emersión, global, local.

KEY WORDS:

Emersion, global, local.

RESUMEN.

En las dos últimas décadas, los dispositivos móviles y las tecnologías de realidad mixta nos han permitido hibridar los espacios digitales y los físicos, creando espacios transcodificados que se retroalimentan mutuamente. La computación ubicua, las tecnologías nómadas y los dispositivos geolocalizados funden la frontera entre el espacio digital global y los espacios físicos locales donde se mueve la persona, lo cual ha transformado la forma en la que ésta experiencia ambos espacios, ya que no accede a ellos a través de una interfaz fija, sino que el espacio físico es ahora una interfaz para el espacio digital. Este artículo pretende analizar esta transcodificación de lo global digital a lo local físico y conceptualizarlo como un fenómeno de emersión. Propone que este giro de dentro hacia fuera cambia la experiencia de la persona y potencia la organización y la creación de estructuras más complejas en el nivel de lo físico. Se propone el concepto de emersión como contrametáfora de inmersión, que consiste en llevar el contenido de un juego digital u otro medio desde dentro hacia fuera, manifestándose en el espacio físico y en la vida de la persona. Además se diferencia del concepto de emergencia o surgimiento, que es la capacidad de un sistema para dar lugar a una novedad impredecible. Este artículo desarrolla el concepto de la emersión en tanto que fenómeno presente en diversos medios, aunque más fácilmente reconocible en los juegos digitales, que es complementario al de inmersión. Para finalizar se presenta la emersión como una transcodificación entre el espacio físico y el espacio digital, así como entre lo virtual y la vida ordinaria.

\section{ABSTRACT.}

In these last two decades, mobile devices and mixed reality technologies have enabled the hybridization of digital and physical spaces, resulting in transcoded spaces that continually feed back each other. Ubiquitous computing, nomadic technologies, and geolocalized devices have blurred the boundary between the global-digital space and the local-physical, and this fact has transformed the way we experience both spaces, since we have ceased to access to the digital using a spatially fixed interface and, therefore, now all the physical space is an interface for the digital one. This paper aims to analyze the transcodification that is happening between the global-digital and the local-physical and seeks to conceptualize it as an emersive phenomenon. This turning from 'the outside in' to the 'inside out' has changed how we experience the digital, as well as enhancing the organization and creation of more complex structures at the level of the physical. Conceptually, emersion is proposed as a countermethaphor of immersion, meaning that the content of a digital game or other medium is carried inside out, taking place in physical space and everyday life. It also differs from the concept of emergence, which means that a given system can develop into an unpredictable novelty. This paper builds the 
concept of emersion as a phenomenon that can be found in various media, although it is more easily recognizable in digital games. Lastly, emersion is presented as a transcodification between physical space and digital space, as well as between virtual and ordinary life.

\section{CONTENIDO.}

\section{INTRODUCCIÓN}

Los dispositivos móviles y las tecnologías de realidad mixta han permitido que el espacio global y el espacio local se superpongan, fundiendo las fronteras entre ambos. Estas tecnologías portátiles no son sólo una interfaz para acceder al espacio digital, que es un conjunto de información interconectada que abarca al mundo entero y no está limitada por la ubicación, sino también una una herramienta para comunicarse con el espacio físico dando lugar a una suerte de espacios transcodificados que Adriana de Souza e Silva adjetiva híbridos:

With no previous connections to the concepts of immersion and virtual reality, mobile digital spaces acquire a completely different meaning to this community of users: instead of focusing on issues such as immersion and identity creation in virtual worlds, users are more likely to be concerned about how their keitai can help them in physical spaces, to find places and friends through location awareness, to buy train tickets, and pay for groceries at the supermarket. (de Souza e Silva, 2006 , pp. 6 y 7)

La movilidad de los dispositivos funde la frontera entre espacios digitales y espacios físicos, dando lugar a un espacio híbrido por el que nos movemos físicamente, pero también a través de estas tecnologías portátiles:

Without the traditional distinction between physical and digital spaces, a hybrid space occurs when one no longer needs to go out of physical space to get in touch with digital environments. Therefore, the borders between digital and physical spaces, which were apparently clear with the fixed Internet, become blurred and no longer clearly distinguishable. (de Souza e Silva, 2006, p. 7)

El problema radica en la intersección entre ambos espacios: el espacio digital y el espacio físico. Al solaparse y confundirse ambos, parecería invalidada la idea de inmersión en tanto que acceso desde el espacio físico al espacio digital. Parece, por lo tanto, que existen otras relaciones entre estos espacios que ya no se pueden describir mediante la inmersión.

Los juegos digitales tradicionales constan de dos ámbitos espaciales diferenciados en cuya confluencia se sitúa el agente: el espacio virtual y el físico. En este modelo, el agente dirige su atención hacia el mundo del juego, sumergiéndose en el entorno virtual que éste le propone. Los elementos de interacción, representación y narración están orientados hacia el espacio virtual, de forma que el agente se sumerge dicho mundo simbólico y espacial para poder jugar. Este sería el paradigma inmersivo, presente en gran parte de los juegos digitales que salen al mercado. La computación ubicua, las tecnologías nómadas y los dispositivos geolocalizados, empero, están cambiando de forma trepidante la relación entre ambos espacios.

Foursquare es un buen ejemplo de este proceso de transformación espacial, sobre todo en las versiones anterior a 2014 cuando abandonó las mecánicas lúdicas de check-in y alcaldías. Su mecánica de juego consistía en marcar el lugar donde uno se encontraba para ganar puntos, descubrir lugares, lograr insignias y alcaldías. La persona que más veces marcaba sobre un lugar en los últimos 60 días se convertía, temporalmente, en el alcalde de dicho lugar. De esa manera, se enriquecía la forma en la que las personas se muevían por el espacio físico, dando lugar, como los llama de Souza e Silva, espacios híbridos.

Otro juego, Ingress -creado por Niantic Labs a iniciativa de Google Inc.- transforma el espacio púbico en un tablero donde suceden acontecimientos virtuales, tales como la creación de portales y campos protectores que protegen a las personas de una área determinada. Ingress no sólo combina el espacio virtual y el espacio físico, sino que los acontecimientos virtuales y los físicos. Para realizar las acciones del juego se tiene que acudir a espacios concretos y por lo tanto el flujo de la acción del juego depende de los movimientos diarios del agente en el espacio físico de la ciudad. Además, el agente realiza desplazamientos que probablemente no haría si no estuviera jugando, adaptando la vida al juego. De esta forma, la idea de que se produce una inmersión en el juego quedaría en entredicho, ya que el juego y la vida se superponen. Después de Ingress, Niantic, Inc desarrolló Pokemon Go, que resultó todavía más mediático, ya que consistía en adaptar la mecánica inmersiva de Pokemon, que ya contaba con millones de seguidores, a la mecánica emersiva desarrollada en Ingress. 


\section{PROPUESTA.}

Por lo tanto, si la relación entre el espacio físico local y el espacio digital global está cambiando en los medios digitales, y muy especialmente en los juegos, cabe hacerse la siguiente pregunta: ¿Qué sucede cuando estos medios se vuelven de dentro hacia fuera de sí mismos? Este artículo propone que el cambio de orientación del medio cambia la experiencia de la persona, que ya no puede definirse mediante la metáfora de la inmersión, en el sentido tradicional atribuido a Janet Murray:

The experience of being transported to an elaborately simulated place is pleasurable in itself, regardless of the fantasy content. We refer to this experience as immersion. Immersion is a metaphorical term derived from the physical experience of being submerged in water. We seek the same feeling from a psychologically immersive experience that we do from a plunge in the ocean or swimming pool: the sensation of being surrounded by a completely other reality, as different as water is from air, that takes over all of our attention, our whole perceptual apparatus. We enjoy the movement out of our familiar world, the feeling of alertness that comes from being in this new place, and the delight that comes from learning to move within it. (...) in a participatory medium, immersion implies learning to swim, to do the things that the new environment makes possible. (Murray, 1997, 98-99).

Esta transcodificación de lo digital hacia lo físico - y cambio de orientación de lo virtual hacia la vida - enfatiza la experiencia de emersión de lo digital-global a lo físico-local. Cuando el medio se vuelve de fuera hacia dentro, parecería no buscar la inmersión en el espacio virtual, sino el acontecimiento en la realidad. Asimismo, parecería que esta emersión de lo digital potencia la organización y la creación de estructuras más complejas en lo físico, que en vez de buscar la solidez, están continuamente emergiendo nuevas formas.

\section{CONTRAMETÁFORA DE INMERSIÓN.}

El concepto de emersión que este artículo propone es, sobre todo, una contrametáfora de inmersión, argumentando que la interfaz entre el espacio físico y el digital no es, como suele ser imaginada, un paso de una única dirección, sino que, por el contrario, permite llevar la acción y el contenido del medio en dos direcciones, de las cuales, la que quizá es más evidente, y en la que suelen centrarse las investigaciones, es la que dirige la acción del juego al espacio virtual, es decir, la inmersión como metáfora de sumergirse completamente en un mundo virtual. La emersión es la metáfora contraria y sirve para dar nombre a lo que sucede cuando la acción del juego sale fuera del contexto virtual y se desarrolla en el espacio físico.

Dansey, en su tesis sobre el beneficio emergente en las experiencias de los juegos pervasivos, explica que en la relación de los juegos con la realidad hay dos direcciones (Dansey, 2013, p. 26). Él lo compara con una puerta abatible que se puede abrir de dos maneras: hacia dentro se "empuja» (push) la acción y el contenido del juego en el entorno virtual; esta sería más propia de los videojuegos tradicionales. La otra es «tirar» (pull) de la acción y el contenido del juego, manifestándose en el espacio físico y en la vida de la persona. Este último corresponde al fenómeno de la emersión, en la que el contenido que el agente «tira» hacia sí es el que emerge del juego hacia el espacio físico.

La metáfora de la inmersión evoca el acto de introducirse en el medio acuático y suele identificarse con la sensación que tiene el agente o usuario de que está inmerso en un espacio virtual, por el que se mueve realizando una representación espacial en su mente. Aplicada en este sentido, la metáfora no es exclusiva de los videojuegos, también aparece en el cine, el teatro y la literatura. Suele considerarse más intensa en los juegos digitales porque necesita cierto esfuerzo e interacción para poder sumergirse en ella. Es lo que Aarseth denomina literatura ergódiga (Aarseth, 1997, p. 28), asegurando que los videojuegos obligan al lector a arriesgarse y tomar decisiones, de forma que éste se siente más involucrado en el espacio ficcional propuesto.

Dado que inmersión es la metáfora que se usa para describir la dirección que va desde el espacio físico al espacio virtual, dibujando la imagen de un buceador que se adentra en el agua, la metáfora más adecuada para describir el fenómeno contrario es la emersión. Por otra parte, en el acto de sumergirse el buceador actúan dos tensiones: una que lo sumerge haciendo que el buceador permanezca inmerso y otra que hace que el buceador emerja. Estas dos tensiones, una que introduce a la persona dentro del contenido del medio y otra que expele el contenido del medio hacia ella, no sólo se dan en los juegos digitales, sino que también se pueden encontrar otros medios. Sucede, por ejemplo, cuando vemos una pintura: por una parte, la persona se introduce a través del espacio pictórico, pero por otra parte, la pintura forma parte del espacio en el que ésta se encuentra creando relaciones espaciales. Estas dos tensiones están armonizadas, por ejemplo, en el trampantojo.

También en una obra de teatro, puesto que al tiempo que lo sumerge en la narrativa, la obra también puede romper la cuarta pared e interactuar con el espectador, hacerle un pregunta, o incluso introducirlo dentro del drama. Estas posibilidades interactivas son utilizadas en ocasiones por la dramaturgia contemporánea. Son especialmente notables las técnicas interactivas que Boal (2002) propone como estrategias teatrales de empoderamiento de las clases oprimidas. En el teatro del oprimido se representan temas de importancia política para los "espect-actores", que pueden salir al escenario y realizar cambios sobre la narración que están representando los actores. Boal describe otras formas de teatro más emersivas, como el teatro invisible, que consiste en representaciones teatrales previamente ensayadas que se representan en espacios públicos sin que los espectadores sepan que lo 
son, haciendo emerger el teatro fuera de su espacio. Emersión es precisamente esa fuerza de transformación individual y social presente en cada medio.

Inmersión y emersión están especialmente presentes en los videojuegos. Se puede ver al observar a un grupo de jugadores que al mismo tiempo que realizan sus acciones en el entorno virtual, se lanzan comentarios el uno al otro: «te vas a enterar» o «no me la vas a hacer como el otro día». Además, en la sala puede haber más espectadores que están viendo y comentando la partida. El juego está dirigido de forma inmersiva, sin embargo, hay una parte de la acción del juego que sale, que se convierte en nexo de unión entre los agentees. Está presente en los comentarios de los agentees, en la corporalidad de estos y en los espectadores que comparten el juego.

Si este juego, en vez de orientar el flujo de la acción hacia el espacio del juego virtual, la orientase hacia el espacio vital del agente, la tensión emersiva se volvería dominante. El flujo de la acción son las alternativas que se le plantean y las decisiones que toma el agente durante el juego, el sinnúmero de movimientos, acciones, operaciones, maniobras y desplazamientos que un agente puede realizar durante una partida. Es lo que pasa en el teatro del oprimido, que el teatro se orienta hacia la persona, está dirigido a sus problemas diarios y su objetivo es empoderarla.

Los juegos digitales emersivos, en vez de sumergir al agente en el espacio virtual, refuerzan la presencia en el entorno cotidiano. La emersión es la contrametáfora de la inmersión en todos estos sentidos, dirige el juego hacia la persona, hacia el espacio que la rodea, hacia sus problemas. Orienta el contenido hacia fuera, en vez de orientarlo hacia dentro, y, como en el teatro invisible, el medio invade la vida del agente y forma parte de su entorno cotidiano.

\section{EMERSIÓN Y EMERGENCIA.}

El termino emersión, del latín emersio, -onis, significa "acción y efecto de emerger un cuerpo de un líquido". (Real Academia Española, 2014). Creado por contraposición a inmersión, del latín immersio, -onis, cuyo sentido es "acción de introducir o introducirse algo en un fluido" (Real Academia Española, 2014). Ambos representan las dos caras de una misma metáfora, utilizada para describir la idea de introducirse en un medio y quedar completamente rodeado por él, o todo lo contrario, la idea de que el contenido de ese medio sale y toma lugar.

Emergencia también se deriva del mismo verbo, emerger, pero como su participio activo, del latín emergens, -entis. Emergente se refiere a aquello "que emerge, que nace, sale y tiene principio de otra cosa» (Real Academia Española, 2014). Este término se desvincula completamente de la metáfora anterior. En los sistemas complejos, emergencia o surgimiento es la capacidad de un sistema para dar lugar a una novedad impredecible. Stephan (1999), en un ensayo sobre las variedades del emergentismo, explica que éste puede ser débil cuando se puede explicar a partir de las propiedades, o fuerte, cuando las nuevas estructuras son independientes de toda observación.

Johnson (2001, p. 87), por otro lado, emplea el término emergencia como un proceso en el que componentes de bajo nivel de una comunidad o sistema se organizan en niveles mayores de sofisticación y consciencia. Según él, la reorganización de los elementos proviene de las bases, más que dirigida como un factor externo. Un ejemplo perfecto para él está en el videojuego Simcity, que trata sobre la creación, gestión y evolución de ciudades.

Sweetser (2006) investiga en su tesis la emergencia como un acercamiento al diseño de mundos de juegos como una alternativa al prediseñado. Propone el uso de autómatas celulares (A.C.) que son modelos matemáticos para crear sistemas dinámicos que evolucionen, creando una colección de objetos que interactúan entre sí de forma espontánea y dan lugar a sistemas naturales con comportamientos emergentes. Sweetser (2006) define un comportamiento emergente como aquel que ocurre cuando reglas independientes interactúan para dar lugar a una conducta que no estaba prevista en el sistema.

Según Dansey (2013, p. 135), que emplea el término en referencia a Johnson, el juego emergente genera beneficios para el espacio físico, haciendo que el juego tienda a la creatividad y la reorganización. La emergencia es muy fuerte en los juegos que crean una cierta ambigüedad en sus normas, permitiendo que se creen nuevas estructuras. Esta clase de emergencia también sucede cuando el flujo del juego sale al espacio físico, ya que para ello se dan combinaciones nuevas y los elementos de bajo nivel, con muy poco nivel de complejidad y estructuración, van organizándose para crear nuevas estructuras y formas de jugar.

De este modo, sintéticamente podemos decir que emergencia y emersión son dos conceptos distintos que aluden a diferentes realidades. Los juegos emersivos pueden tener características emergentes, es decir, no previstas, y potenciar la creación de nuevos sistemas. Si emergencia se refiere al proceso por el cual en un sistema genera nuevas estructuras complejas que no estaban previstas, emersión hace referencia al proceso en el que el flujo de la acción emerge hacia el espacio físico, tanto en el sentido de realidad cotidiana como de espacio no computacional. Es un cambio en la dirección del flujo de juego, que de ser de fuera hacia dentro, pasa a ser de dentro hacia fuera. 


\section{ORIENTACIÓN DEL MEDIO DE DENTRO HACIA FUERA.}

Por lo tanto, parece que la relación entre inmersión y emersión es una cuestión de orientación del medio. Un medio puede estar orientado hacia dentro del sistema simbólico y representacional creado por él mismo como lo están una telenovela, un libro de ficción, una obra de teatro convencional o un videojuego. O puede orientar el sistema simbólico y representacional del medio hacia fuera de sí mismo, como, por ejemplo, sucede en un noticiero de televisión, un ensayo literario, o un medio serio.

Si en la inmersión el sujeto se abstrae del medio que nos rodea, en la emersión, el sujeto se reconoce a sí mismo en el entorno físico. En este último, el contenido del medio, el espacio virtual del medio y los retos éste que presenta están orientados de alguna manera al espacio físico y a la realidad ordinaria, es decir, están orientados de dentro hacia fuera. Un buen ejemplo es la conducción guiada por navegador, motivada por llegar a un lugar concreto en el espacio físico, solucionando los problemas del tráfico en las carreteras. A pesar de tener una representación virtual, él conductor se siente presente en el espacio físico, en el que tiene agencia y donde sus actos tienen repercusión.

Este cambio de orientación de dentro hacia fuera tiene los dos aspectos: el cultural y el tecnológico. Respecto al cultural, el cambio de orientación se presenta como consciencia plena de que por una parte, el medio se expande más allá del espacio contractual de representación. En cuanto al tecnológico, es una representación del medio en el espacio físico. Pero la emersión no sólo se da en forma de realidad aumentada, la realidad virtual también tiene contornos borrosos, como muestran Gemeinboeck y Blach (2005) en Uzume y Maᄉa - Veil of Illusion, que son dos instalaciones basadas en CAVE. Estas dos instalaciones establecen un límite líquido y poroso entre el espacio virtual y el físico, creando un espacio intermedio entre lo virtual y lo físico.

Both, Uzume and Maᄉa produce a reality that can never be controlled or be reproduced. Rather, they create a space inbetween the real and the virtual, perforating and interlacing the two: it is to be (bodily) mediated and negotiated and always unfolds inthe present. (Gemeinboeck \& Blach, 2005, p. 87).

La emersión es un fenómeno presente en diversos medios de manera complementaria a la inmersión. Valga de ejemplo cómo ciertas vanguardias artísticas del siglo XX tuvieron la ambición de influir en la realidad, como el movimiento artístico Fluxus, que perseguía la fusión entre arte y vida. También el medio cinematográfico se ha utilizado como una forma de educación y propaganda, haciendo que los modelos sociales se vean influidos por él.

Este proceso de emersión y cambio de orientación de dentro hacia fuera también sucedió en Internet. Al principio estaba lo que después se conoció como web 1.0, una interfaz que permitía la navegación hipertextual, y la creación de mapas y mesetas de conocimiento pero de modo estático y poco interactivo. La web 2.0 dio un giro a Internet, orientándolo hacia los usuarios. Entonces comenzaron a crearse las redes sociales y la web se convirtió en el medio global de interacción. En cierta manera, el cambio en la interfaz del Internet orientó su contenido hacia la vida y hacia el espacio físico. Internet pasó de ser un libro hipertextual a una extensión de la vida misma.

En los medios digitales el cambio de orientación se ha producido debido a diversas razones: las tecnologías de realidad mixta se han abaratado; desde el 2008 los dispositivos ubicuos y geolocalizados como lo teléfonos inteligentes se han vuelto de uso común y se ha experimentado con nuevos usos y propósitos para los medios digitales. Todo esto ha hecho posible el cambio de orientación de dentro hacia fuera, creando un fenómeno que tiene una entidad propia

\section{TRANSCODIFICACIÓN DE LO GLOBAL DIGITAL EN LO LOCAL FÍSICO.}

En los años 80 la sociedad empezó a ser consciente de una realidad que iba cambiar la concepción del espacio global. Se trataba de una realidad configurada por la conexión en red de todos los ordenadores del mundo que tendría como consecuencia un suerte de lugar inmaterial al que se podría acceder desde diferentes puntos locales. Al principio se dio cuenta de ello en la ciencia ficción, identificándolo con el término de ciberespacio. Es especialmente reseñable la novela Neuromante de William Gibson (1997 [1984]), en la que lo describe como una "representación gráfica" de datos concretos al mismo tiempo que una "alucinación consesual":

El ciberespacio. Una alucinación consensual experimentada diariamente por billones de legítimos operadores, en todas las naciones, por niños a quienes se enseña altos conceptos matemáticos... Una representación gráfica de la información abstraída de los bancos de todos los ordenadores del sistema humano. Una complejidad inimaginable. Líneas de luz dispuestas en el no-espacio de la mente, agrupaciones y constelaciones de datos..., el propio terreno de lo virtual, donde todos los medios se juntan (fluyen) y nos rodean. (Gibson, 1997 [1984], p. 14)

El ciberespacio tenía sus propios códigos, diferentes de los del espacio físico, y estaban basados en la representación gráfica que da una forma comprensible para el ser humano a estas "constelaciones" o bases de datos. Al principio este espacio cibernético y los espacios locales desde donde se accedía a él se imaginaban como algo completamente separado e incompatible, puesto que uno era una infinita representación mental de datos abstractos, y el otro, en cambio, era físico y limitado. 
Sin embargo, a partir de la irrupción de los dispositivos portátiles y geolocalizados, estos espacios empezaron a hibridarse y compartir códigos. El ciberespacio dejó de estar contenido en sí mismo y empezó a verterse sobre el espacio físico, produciendo un fenómeno de emersión, una orientación del medio de dentro hacia fuera. La persona ya no necesitaba entrar en el otro espacio, no tenía cambiar de códigos para acceder a lo digital global, sino que lo digital global estaba en todas partes. Era como sí la Red se hubiera tornado de hacia fuera de sí mismo. William Gibson, 26 años después de publicar Neuromante, reflexionó sobre esta idea:

Cyberspace, not so long ago, was a specific elsewhere, one we visited periodically, peering into it from the familiar physical world. Now cyberspace has everted. Turned itself inside out. Colonized the physical. Making Google a central and evolving structural unit not only of the architecture of cyberspace, but of the world. This is the sort of thing that empires and nation-states did, before. But empires and nation-states weren't organs of global human perception. They had their many eyes, certainly, but they didn't constitute a single multiplex eye for the entire human species. (Gibson, 2010, p. 23)

Ocurrió una suerte transcodificación de ambos espacios, el global-digital y el local-físico. El espacio de lo digital comenzó a adquirir códigos propios de lo físico, como la localización y la espacialidad, haciendo que el lugar desde que se accede afecte al espacio al que se accede, y los resultados de una búsqueda concreta, por ejemplo, no sean iguales en un lugar o en otro. Se puede observa también en que las relaciones generadas en las redes sociales, que son un código propio de lo local-físico, son hoy un código esencial de lo global-digital.

Este fenómeno también ocurrió en sentido contrario y sobre lo local físico empezó a crearse una capa de datos digitales que completan y amplían el espacio local-físico. Hasta tal punto hemos completado la hibridación, que no es fácil decir donde termina uno y dónde comienza el otro. Un acontecimiento local puede pasar a ser global en apenas unos segundos y viceversa, lo que ocurren en el espacio virtual afecta a lo local muy rápidamente.

Los códigos de lo global y lo local se retrolimentan hasta fundirse. La emersión, o la orientación del medio de dentro hacia fuera, es lo que ha permitido esta transcodificación en la que lo global-digital tiene sentido en tanto que emerge al espacio local-físico, que a su vez depende de los códigos de lo global para manifestarse como fenómeno. Es el caso de los retos (challenges) en las redes sociales, como el Ice Bucket Challenge o el Mannequin Challenge, que surgen de publicaciones en Internet que son rápidamente replicadas por otros muchos usuarios, llegando a los medios de comunicación de masas, habitualmente, cuando son realizados por personas famosas. El cambio constante, la reestructuración, la modularidad o la repetición son códigos de lo global-digital que han tomado lugar en lo local, ocasionando una emergencia fluida que permite reorganizaciones constantes y cada vez más complejas, en las que es difícil mantener estructuras fijas y sólidas.

\section{CONCLUSIONES.}

Este artículo pretendía investigar qué ocurre cuando los medios digitales se vuelven hacia fuera de sí mismos, y particularmente, cuando el espacio global-digital que se conforma mediante la conexión en Red se vierte en lo local-físico. Aunque solemos entender la relación entre las personas y los medios como una inmersión, sucede también lo contrario, una emersión: el contenido emerge hacia el espacio local-físico y la persona no tiene la sensación de estar inmersa en un no-espacio o representación espacial, sino en el suyo propio. Emersión es un concepto diferente de emergencia, y se refiere a cuando un medio se torna de dentro hacia fuera de sí mismo, mientras que emergencia se refiere a la capacidad de estos para evolucionar a sistemas más complejos.

Hemos observado que el espacio global-digital, al que antes se accedía desde una estación fija u ordenador conectado a Internet, cada vez está más hibridado con el espacio físico-local. Ahora ambos espacios comparten códigos, pero es una transcodificación producida por la emersión, por la capacidad del medio de salir fuera de sí mismo. Futuros desarrollos de esta investigación pasan por estudiar las relaciones entre inmersión y emersión que se están produciendo en medios con lenguajes específicos, tales como los videojuegos o el cine.

\section{FUENTES REFERENCIALES.}

AARSETH, Espen. Cybertext: perspectives on ergodic literature. Seattle : The Johns Hopkins University Press,1997, 216 pp. ISBN 0801855780 .

BOAL, Augusto. Juegos para actores y no actores: teatro del oprimido. Barcelona : Alba Editorial, 2002. ISBN 978-84-8428-134-4.

DANSEY, Neil. A Grounded Theory of emergent benefit in pervasive game experiences. [En línea]. Tesis Doctoral. Universidad de Portsmouth, 2013. 262 pp. Disponible en: http://eprints.port.ac.uk/12470/1/NeilDanseyThesis.pdf 

http://dx.doi.org/10.4995/ANIAV.2017.4954

DE SOU A E SILVA, Adriana. From cyber to hybrid: Mobile technologies as interfaces of hybrid spaces. Space and Culture. 2006. Vol. 9, no. 3, pp. 261-278

GEMEINBOECK, Petra y BLACH, Roland. Spacing the Boundary: An Exploration of Perforated Virtual Spaces. PsychNology Journal 2005. Vol. 3, no. 1, pp. 74-89.

GIBSON, William. Google's Earth. The New York Times. Nueva York, 1 Septiembre 2010. pp. A23.

GIBSON, William. Neuromante. Barcelona : Ediciones Minotauro, 1997 [1984]. 208 pp. ISBN 9788445076620.

JOHNSON, Steven Berlín. Emergence: The Connected Lives of Ants, Brains. Nueva York : Scribner, 2001. 288 pp. ISBN 0-684-86875.

MURRAY, JH. Hamlet on the holodeck: The future of narrative in cyberspace. Nueva York: MIT Press, 1997. 336 pp.

REAL ACADEMIA ESPAÑOLA. El Diccionario de la lengua española (23a edición) [En línea]. 2014. Disponible en: http://dle.rae.es

STEPHAN, Achim. Varieties of Emergentism. Evolution and Cognition [En línea].1999. Vol. 5, no. 1, pp. 49-59. Disponible en: http://www.brynmawr.edu/biology/emergence/stephan.pdf

SWEETSER, Penelope. An Emergent Approach to Game Design - Development and Play [En línea]. The University of Queensland, 2006. [Acceso 30 marzo de 2016] Disponible en: http://eprints.qut.edu.au/46352/1/Sweetser_GCAP08.pdf 